\title{
Increased serum chemerin level to predict early onset of aortic valve stenosis
}

\author{
JURIS LURINS ${ }^{1}$, DACE LURINA ${ }^{2}$, PETERIS TRETJAKOVS ${ }^{3}$, VITOLDS MACKEVICS ${ }^{4}$, \\ AIVARS LEJNIEKS ${ }^{4}$, VENERANDO RAPISARDA ${ }^{5}$ and VINCENZO BAYLON ${ }^{6}$
}

\author{
${ }^{1}$ Department of Doctoral Studies, Riga Stradins University, Riga, LV 1007; ${ }^{2}$ Zemgale Health Centre, Jelgava, LV 3001; \\ ${ }^{3}$ Faculty of Medicine, Department of Human Physiology and Biochemistry, Riga Stradins University, Riga, LV 1007; \\ ${ }^{4}$ Faculty of Medicine, Department of Internal Diseases, Riga Stradins University, Riga, LV 1002, Latvia; \\ ${ }^{5}$ Department of Clinical and Experimental Medicine, Occupational Medicine, University Hospital \\ 'Policlinico-Vittorio Emanuele', University of Catania, I-95123 Catania, Italy; \\ ${ }^{6}$ Newton Lewis Institute-ISR - Life Science Park, San Gwann 3000, Malta
}

Received October 16, 2017; Accepted October 23, 2017

DOI: $10.3892 / b r .2017 .1010$

\begin{abstract}
Inflammation appears to be the cause of aortic valve (AoV) stenosis and identification of predictive biomarkers is therefore imperative. The aim of the current study was to evaluate the potential role of serum chemerin and fibroblast growth factor-21 (FGF-21) in the pathogenesis of the disease. A total of 102 patients were selected based on certain criteria and divided into an aortic stenosis group and a control group. Patients with AoV stenosis were subdivided into three groups depending on the severity according to the echocardiography criteria: Aortic jet velocity, Vmax (m/ sec); mean pressure gradient, $\mathrm{PG}(\mathrm{mmHg})$; aortic valve area (AVA), $\mathrm{cm}^{2}$; and indexed AVA, $\mathrm{cm}^{2} / \mathrm{m}^{2}$. Patients were graded as: Severe: Vmax $>4 \mathrm{~m} / \mathrm{sec}, \mathrm{PG}>40 \mathrm{mmHg}$, AVA $<1.0 \mathrm{~cm}^{2}$, indexed AVA <0.6; moderate: Vmax 3.0-4.0 m/sec, PG 20-40 mmHg, AVA 1.0-1.5 cm², indexed AVA 0.60-0.85; mild: Vmax 2.5-2.9 m/sec, PG $<20 \mathrm{mmHg}$, AVA $>1.5 \mathrm{~cm}^{2}$, indexed AVA $>0.85$. ELISA was used for the detection of chemerin and FGF-21. Post-hoc analysis with Tukey's correction was performed. The highest chemerin levels were found in mild and moderate AoV stenosis and decreased along with the grade of severity, compared with the control group. The FGF-21 level was increased in all the stenosis groups, reaching the highest level at severe stenosis. Receiver-operating
\end{abstract}

Correspondence to: Dr Juris Lurins, Department of Doctoral Studies, Riga Stradins University, 16 Dzirciema Street, Riga, LV 1007, Latvia

E-mail: jl.cardio@gmail.com

Abbreviations: CMKLR1, chemokine-like receptor 1; ChemR23, chemerin receptor 23; FGF-21, fibroblast growth factor-21; AoV, aortic valve; $\mathrm{CRP}, \mathrm{C}$-reactive protein

Key words: aortic valve stenosis, chemerin, FGF-21, biomarkers characteristic analysis of chemerin in all the AoV stenosis groups without grading the severity included, area under the curve $(\mathrm{AUC})=0.76 ; 0.70-0.80=$ fair; $\mathrm{P}<0.001$ and for mild AoV stenosis was $\mathrm{AUC}=0.82 ; 0.80-0.90=$ good; $\mathrm{P}<0.001$. In conclusion, chemerin is a good diagnostic biomarker for mild AoV stenosis, while FGF-21 is a moderate diagnostic marker.

\section{Introduction}

Aortic valve stenosis (AoV), a global cardiovascular health concern, is the most frequent valve disease, the third most frequent cardiovascular disease after arterial hypertension and coronary heart disease, and the most widespread indication for surgical valve replacement (1-3). Data of systemic studies showed that calcific AoV sclerosis is present in approximately $9 \%$ of patients aged 54 and above, to $42 \%$ at age 81 . In $1.8-1.9 \%$ of patients, AoV sclerosis progresses to clinical calcific AoV stenosis. These patients have increased risk of coronary heart disease $(68 \%)$, myocardial infarction (27\%), cardiac death (69\%), and risk of other etiology death, which makes calcific AoV stenosis a significant risk factor for morbidity and mortality in the age groups mentioned (4). If the AoV is not replaced, $75 \%$ of patients succumb in 3 years and $50 \%$ above the age of 65 years succumb within 2 years after the occurrence of symptoms such as chest pain, syncope, and heart failure (5). Historically, AoV stenosis was considered a degenerative disease related to aging, and wear and tear of the valve. Nevertheless, there is no distinct answer as to whether it is the cause of AoV stenosis onset and progression, as many elderly people do not suffer from this disease (6).

Regarding pathogenetic aspects of AoV stenosis, valves are made of specialized cells with distinct functions: Valvular endothelial cells, valvular interstitial cells, and the extracellular matrix, which consists of collagen, elastin, and glycosaminoglycans. Valvular interstitial cells are of five different phenotypes with distinct biological functions; embryonic endothelial and mesenchymal progenitor cells, quiescent, 
activated, progenitor, and osteoblast-like valvular interstitial cells, with the latter contributing to the calcification of AoV (1).

Calcification at the tissue level is the most common finding in AoV stenosis (7). There are findings to prove the active inflammation process, such as the increased expression of C-reactive protein (CRP), increased temperature in stenotic valve leaflets, and a special non-invasive method combining positron emission tomography and computed tomography using a ligand 18 fluordesoxyglucose, therefore proving the activity of macrophages in stenotic valve leaflets $(8,9)$.

Chemerin is an adipokine known as a ligand for $\mathrm{G}$ proteincoupled chemokine-like receptor 1 , also known as chemerin receptor 23, which was first identified in macrophages and dendritic cells (10-12). The main source of chemerin is white adipose tissue, but a high concentration was also found in the liver $(13,14)$, lower lungs, brown adipose tissue, heart, ovaries, kidneys, skeletal muscle, and pancreas (15). The circulating chemerin level strongly correlates with the level of inflammatory markers such as tumor necrosis factor (TNF)- $\alpha$, interleukin (IL)-6, high-sensitivity CRP, resistin, and leptin $(15,16)$ in humans and animals (17-19). Chemerin regulates the inflammatory process by promoting the migration of macrophages to certain tissues such as adipose tissue (20).

It is clear that chemerin has a role in inflammatory processes, but there is still no consensus whether it is a proor anti-inflammatory activity (21-27). Eisinger et al showed that chemerin has no positive correlation with the severity of inflammation (17). A link between the severity grade of liver cirrhosis and the severity of chronic hepatitis $\mathrm{C}$ and plasma chemerin levels has been investigated, and a negative correlation was found in both cases, which leads to the conclusion that chemerin may have a protective role in the inflammation process $(17,28)$. Studies revealed that various chemerin fragments with pro- and anti-inflammatory activity are produced depending on predominating proteases in the inflammatory process $(29,30)$.

Fibroblast growth factor-21 (FGF-21) has an important role in cardiovascular diseases. It is a protein secreted in the liver, skeletal muscle, adipose tissue, and pancreas (31). It has been found that the FGF-21 serum level is increased in patients with abnormal lipid profiles, obesity, metabolic syndrome, reduced glucose tolerance, diabetes type 2 , hypertension, non-alcoholic fatty liver disease, and coronary artery disease (31-33). Similarly, the FGF-21 level is increased in patients with coronary heart disease and atherosclerotic plaques of the carotid arteries; thus, FGF-21 can be used as a biomarker in atherosclerotic diseases (34-36).

FGF-21 has been shown to reduce IL-6, IL- $\beta$, and TNF- $\alpha$ production, which assume the main role in the pathogenesis of inflammation (37).

Findings support the protective role of FGF-21's in different cardiovascular diseases, such as atrial fibrillation, myocardial infarction, coronary artery disease, and hypertension. Han et al stated that the FGF-21 level was markedly higher in patients with atrial fibrillation compared to a control group (38).

It is also stated that cardiomyocytes are capable of secreting FGF-21 as a response to various stress factors, such as cardiac hypertrophy or myocardial infarction (39-42). FGF-21 has an autocrine effect on preventing cardiac inflammation, development of hypertrophy, apoptosis of cardiomyocytes, and the emergence of oxygen-free radicals, as well as promoting the oxidation of fatty acids (42).

The aim of the present study was to evaluate serum chemerin and serum FGF-21 as possible biomarkers of AoV stenosis and to study inflammation in the pathogenesis of AoV stenosis.

\section{Patients and methods}

Study population. This prospective study was conducted from 2013 to 2016 in different hospitals in Latvia. The study protocol had prior approval from the Riga Stradins University (Riga, Latvia) Ethics Committee on research on humans and the study protocol conforms to the ethical guidelines of the 1975 Declaration of Helsinki.

A total of 102 patients were selected on the basis of inclusion and exclusion criteria and divided in two core groups, a control and an AoV stenosis group. The control group included patients without AoV stenosis aged from 55 to 80 years, which complies with the average age of AoV stenosis patients according to the Guidelines on the Management of Valvular Heart Disease 2012 of the European Society of Cardiology (43). Written informed consent was obtained from each patient included in the study. Patients were included in the control group according to echocardiographically confirmed results of a healthy AoV. Exclusion criteria in the two groups were obesity, systemic connective tissue, infectious and oncological diseases, diabetes, thyroid dysfunction and arterial hypertension, atherosclerotic coronary artery disease, heart failure, myocardial infarction, cerebral infarction and transient ischemic attack. Exclusion criteria in AoV stenosis group were patients with congenital AoV stenosis and rheumatic AoV stenosis.

AoV stenosis assessment. Patients were examined echocardiographically and the data were archived using GE VIVID 7 Dimension and Philips IE 33, both from KPI Healthcare (Yorba Linda, CA, USA). Each echocardiography examination was evaluated by two professionals.

Patients with AoV stenosis were subdivided into three groups (Table I) depending on the severity grade according to the echocardiography criteria: aortic jet velocity (Vmax) (m/sec); mean pressure gradient, $\mathrm{PG}(\mathrm{mmHg})$; aortic valve area, AVA $\left(\mathrm{cm}^{2}\right)$ and indexed AVA $\left(\mathrm{cm}^{2} / \mathrm{m}^{2}\right)$. Data were graded as: Severe: Vmax $>4 \mathrm{~m} / \mathrm{sec}, \mathrm{PG}>40 \mathrm{mmHg}$, AVA $<1.0 \mathrm{~cm}^{2}$, indexed AVA <0.6; moderate: Vmax 3.0-4.0 m/sec, PG 20-40 mmHg, AVA 1.0-1.5 cm², indexed AVA 0.60-0.85; mild: Vmax 2.5-2.9 m/sec, PG $<20 \mathrm{mmHg}$, AVA $>1.5 \mathrm{~cm}^{2}$, indexed AVA $>0.85$.

Biochemical analysis. Venous blood samples were taken and serum was obtained in accordance with the serum preparation instructions. Serum samples were stored at $-80^{\circ} \mathrm{C}$ and were available in 102 patients. We obtained laboratory analyses for all the patients at the Biochemistry Laboratory of the Riga Stradins University, including chemerin and FGF-21 with the ELISA method, using human Chemerin ELISA (cat. no. EZHCMRN-57K) and human FGF-21 ELISA (cat. no. EZHFGF21-19K) kits, both produced by EMD Millipore (Billerica, MA, USA). Results were detected using a TECAN Infinite 200 PRO multimode reader (Tecan Group, Ltd., 
Table I. Baseline characteristics of the patients.

\begin{tabular}{|c|c|c|c|c|}
\hline Characteristics & $\begin{array}{c}\text { Control } \\
n=50\end{array}$ & $\begin{array}{l}\text { AoV mild stenosis } \\
n=18\end{array}$ & $\begin{array}{l}\text { AoV moderate stenosis } \\
\mathrm{n}=19\end{array}$ & $\begin{array}{c}\text { AoV severe stenosis } \\
n=15\end{array}$ \\
\hline \multicolumn{5}{|l|}{$\operatorname{Sex}(\%)$} \\
\hline Male & $11(22.0)$ & $2(11.1)$ & $8(42.1)$ & $7(46.7)$ \\
\hline Female & $39(78.0)$ & $16(88.9)$ & $11(57.9)$ & $8(53.3)$ \\
\hline Age, mean $\pm \mathrm{SD}$ & $65.18(9.74)$ & $70.53(6.08)$ & $72.16(8.20)$ & $65.27(8.13)$ \\
\hline P-value vs. control & & $\mathrm{P}=0.127$ & $P=0.012$ & $P>0.999$ \\
\hline $\mathrm{BMI}^{\mathrm{a}}$, mean $\pm \mathrm{SD}$ & $26.04(4.31)$ & $27.39(3.10)$ & $25.81(4.58)$ & $27.40(3.18)$ \\
\hline P-value vs. control & & $\mathrm{P}=0.399$ & $\mathrm{P}=0.682$ & $\mathrm{P}=0.869$ \\
\hline $\mathrm{LDL}^{\mathrm{b}}, \mathrm{mmol} / 1$ mean $\pm \mathrm{SD}$ & $3.28(1.18)$ & $3.05(0.97)$ & $2.59(0.92)$ & $3.10(1.12)$ \\
\hline P-value vs. control & & $\mathrm{P}>0.999$ & $\mathrm{P}=0.057$ & $\mathrm{P}>0.999$ \\
\hline Triglycerides $\mathrm{mmol} / \mathrm{l}$, mean $\pm \mathrm{SD}$ & $1.47(0.71)$ & $1.64(0.84)$ & $1.11(0.56)$ & $1.27(0.57)$ \\
\hline P-value vs. control & & $\mathrm{P}=0.406$ & $\mathrm{P}=0.178$ & $\mathrm{P}=0.406$ \\
\hline Total cholesterol mmol/l, mean $\pm \mathrm{SD}$ & $5.49(1.28)$ & $5.01(1.34)$ & $4.21(1.18)$ & $4.68(1.08)$ \\
\hline P-value vs. control & & $\mathrm{P}=0.056$ & $\mathrm{P}=0.001$ & $P=0.016$ \\
\hline hs-CRP $\mathrm{mg} / \mathrm{l}$, & 5.03 & 4.69 & 5.27 & 3.7 \\
\hline Median (IQR) & $(2.12-14.23)$ & $(3.42-7.35)$ & $(1.71-11.14)$ & $(1.56-11.9)$ \\
\hline P-value vs. control & & $\mathrm{P}>0.999$ & $\mathrm{P}>0.999$ & $\mathrm{P}>0.999$ \\
\hline${ }^{\mathrm{d}} \mathrm{SV} \mathrm{ml}$, mean $\pm \mathrm{SD}$ & $81.86(20.11)$ & $73.63(18.92)$ & $77.72(18.65)$ & $75.93(15.49)$ \\
\hline P-value vs. control & & $\mathrm{P}=0,299$ & $\mathrm{P}=0.501$ & $\mathrm{P}=0.501$ \\
\hline${ }^{\mathrm{e}} \mathrm{EF} \%$, mean $\pm \mathrm{SD}$ & $62.22(6.35)$ & $57.16(9.23)$ & $61.78(8.23)$ & $56.13(7.84)$ \\
\hline P-value vs. control & & $P=0.014$ & $\mathrm{P}=0.291$ & $\mathbf{P}=\mathbf{0 . 0 0 7}$ \\
\hline
\end{tabular}

${ }^{a} \mathrm{BMI}$, weight in kilograms divided by the square of the height in meters; ${ }^{\mathrm{b}} \mathrm{LDL}$, low-density lipoprotein cholesterol; hs-CRP ${ }^{\mathrm{c}}$, is high-sensitivity C-reactive protein; dSV, stroke volume, measured by left ventricular outflow method; 'EF, ejection fraction, measured by Simpson's method. Bold, statistically significant.

Table II. Sensitivity and specificity of the chemerin and FGF-21 in patients with aortic valve stenosis (including all severity degrees of stenosis).

\begin{tabular}{|c|c|c|c|c|c|c|c|c|}
\hline Biomarker & AUC $(95 \%$ CI) & P-value & Cut-off value & $\mathrm{Sp} \%$ & $\mathrm{Se} \%$ & NPV \% & PPV \% & Accuracy $\%$ \\
\hline Chemerin (ng/ml) & $0.76(0.67-0.85)$ & $<0.001$ & 38.60 & 55 & 80 & 72.2 & 63.6 & 67.5 \\
\hline $\mathrm{FGF}(\mathrm{pg} / \mathrm{ml})$ & $0.67(0.56-0.77)$ & 0.003 & 309.83 & 67 & 61.5 & 61.5 & 66.6 & 64.2 \\
\hline
\end{tabular}

AUC, area under the curve; Sp, specificity; Se, sensitivity; NPV, negative predictive value; PPV, positive predictive value; FGF, fibroblast growth factor.

Mannedorf, Switzerland). The procedure was carried out in accordance with the manufacturer's instructions.

Statistical analysis. The data were analyzed using IBM SPSS version 23.0 (IBM Corp., Armonk, NY, USA). Descriptive statistics were performed according to the data type. The results for age, body mass index, low-density lipoprotein cholesterol, total cholesterol, triglycerides and ejection fraction, as well as stroke volume are expressed as mean (M) and standard deviation $( \pm \mathrm{SD})$. Results for high-sensitivity CRP are expressed as median and interquartile range.

Laboratory parameters between the groups were compared using analysis of covariance (ANCOVA) while controlling age as a covariate. Tukey's test was used as a post-hoc test to determine any significant differences between the groups. The performance of the chemerin and FGF-21 was assessed using receiver-operating characteristic (ROC) curves, sensitivity, specificity, and negative and positive predictive values. The P-value was reported for the area under the curve (AUC) for the best cut-off level. Diagnostic tests were assessed by this classification: $0.90-1=$ excellent; $0.80-0.90=$ good; $0.70-0.80=$ fair; 0.60-0.70=poor; and 0.50-0.60=fail. A two-tailed P-value less than 0.05 was considered to indicate a statistically significant difference.

\section{Results}

Patient characteristics. Patient characteristics are provided in Table I and the results of laboratory measurements in Tables II and III. A total of 102 patients were included and divided into 
Table III. Sensitivity and specificity of chemerin and FGF-21 in the mild AoV stenosis group.

\begin{tabular}{lcccccccc}
\hline Biomarker & AUC $(95 \% \mathrm{CI})$ & P-value & Cut-off value & Sp $\%$ & Se $\%$ & NPV $\%$ & PPV \% & Accuracy $\%$ \\
\hline Chemerin $(\mathrm{ng} / \mathrm{ml})$ & $0.82(0.70-0.95)$ & $<0.001$ & 43.12 & 69 & 87 & 75.5 & 71.9 \\
FGF $(\mathrm{pg} / \mathrm{ml})$ & $0.66(0.51-0.81)$ & 0.04 & 283.78 & 61 & 75 & 64.4 & 65.4 & 68
\end{tabular}

AUC, area under the curve; Sp, specificity; Se, sensitivity; NPV, negative predictive value; PPV, positive predictive value; FGF-21, fibroblast growth factor-21; CI, confidence interval; AoV, aortic valve.

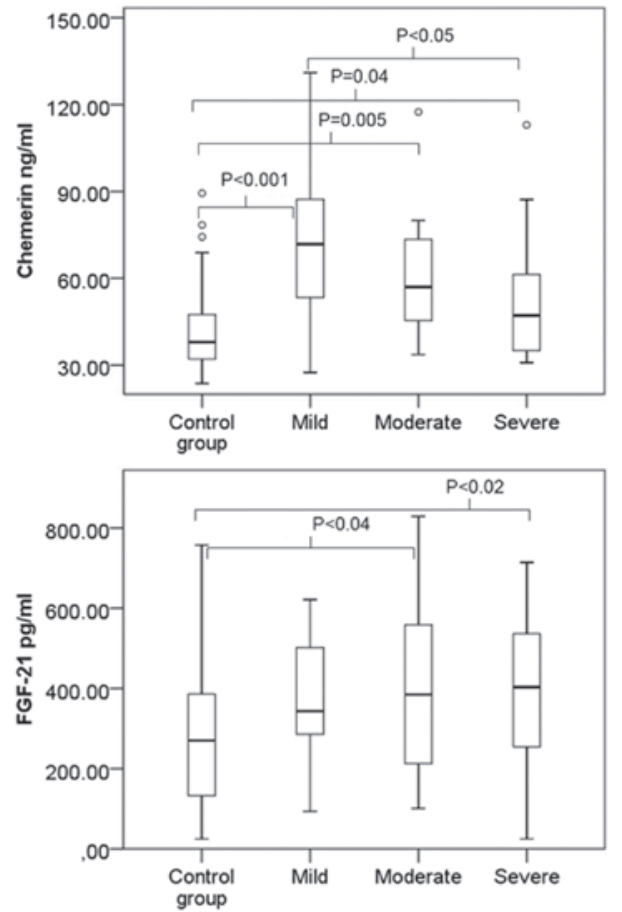

Figure 1. Box and whisker plot of chemerin and FGF-21 in the control and AoV stenosis groups. The highest plasma chemerin level was found in mild AoV stenosis. The highest plasma FGF-21 level was found in severe AoV stenosis. FGF-21, fibroblast growth factor-21; AoV, aortic valve.

three AoV stenosis severity groups and a control group: A total of 18 patients had mild, 19 moderate, and 15 severe AoV stenosis, and there were 50 patients in the control group. The average age of the patients in the aortic stenosis groups and the control group were similar, and the mean level of body mass index did not differ between the groups.

Chemerin and FGF-21 level differences between the patient groups. A box and whisker plot of chemerin and FGF-21 for the control and AoV stenosis groups is shown in Fig. 1. The mean chemerin and FGF-21 levels had a statistically significant difference in the study groups (ANCOVA, $\mathrm{P}<0.001$, and $\mathrm{P}=0.03$; age as covariate). To evaluate a difference between the groups, a post-hoc analysis with Tukey's correction for chemerin was performed. Results showed that the control group had a statistically significant difference with the mild AoV stenosis group $(\mathrm{P}<0.001)$, with the moderate AoV stenosis group $(\mathrm{P}=0.005)$, and with the severe AoV stenosis group $(\mathrm{P}=0.04)$. The mild AoV stenosis group had a statistically significant difference with the severe AoV stenosis

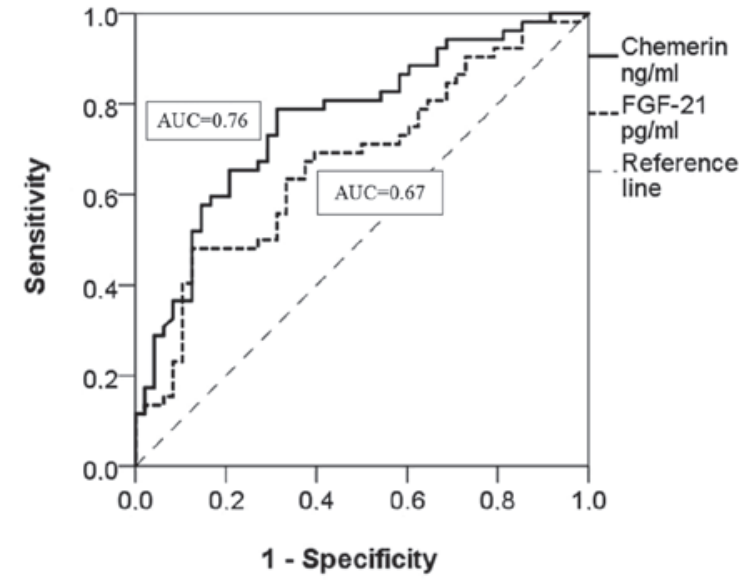

Figure 2. ROC analysis of chemerin and FGF-21 as diagnostic markers of AoV stenosis in the control group vs. all stenosis groups. Chemerin: $\mathrm{AUC}=0.76$ and precision category, fair, a moderate diagnostic biomarker for AoV stenosis; FGF-21: $\mathrm{AUC}=0.67$ and precision category, poor, weak, biomarker for AoV stenosis. AUC, area under the curve; ROC, receiver-opera ting characteristic; FGF-21, fibroblast growth factor-21.

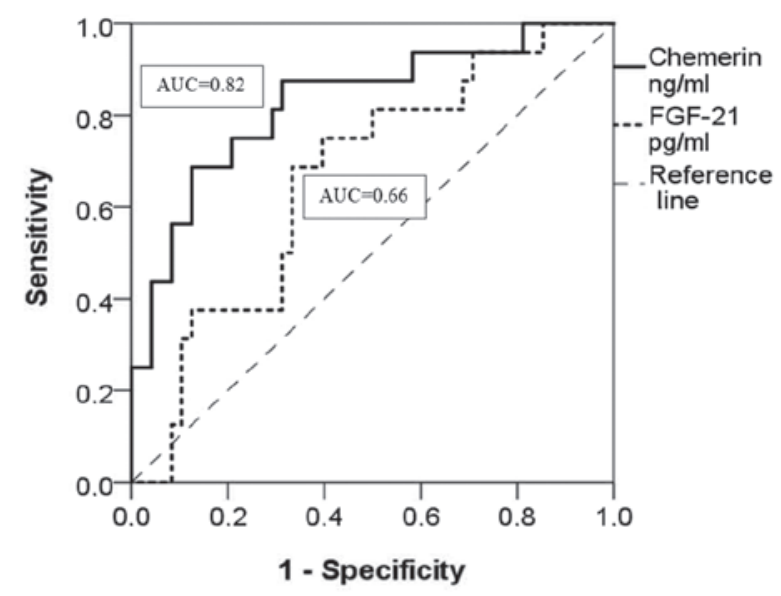

Figure 3. ROC analysis of chemerin and FGF-21 as diagnostic markers of the AoV stenosis - control group vs. the mild AoV stenosis group. Chemerin: $\mathrm{AUC}=0.82$ and precision category, good, a good diagnostic biomarker for AoV stenosis; FGF-21: AUC=0.66 and precision category, poor, weak, biomarker for AoV stenosis. AUC, area under the curve; ROC, receiver-opera ting characteristic; FGF-21, fibroblast growth factor-21

group $(\mathrm{P}<0.05)$, while the moderate and severe AoV stenosis groups had no statistically significant difference $(\mathrm{P}>0.05)$. Post-hoc analysis for FGF-21 revealed that the control group with moderate and severe stenosis groups had a statistically 
significant difference $(\mathrm{P}<0.05)$, but for the rest of the groups, there was no statistically significant difference $(\mathrm{P}>0.05)$ (Fig. 1).

The chemerin serum level increased in all three stenosis groups. The highest chemerin levels were found in mild and moderate AoV stenosis and decreased along with the grade of severity, compared to the control group (Fig. 1). The FGF-21 level increased in all of the stenosis groups, but the FGF-21 level in serum increased along with the AoV stenosis severity grade, reaching the maximum for severe stenosis (Fig. 1).

Predictive values of chemerin as biomarker. The ROC analysis showed that chemerin is a moderate diagnostic marker in all AoV stenosis groups without grading the severity ( $\mathrm{AUC}=0.76$; 0.70-0.80=fair; $\mathrm{P}<0.001$ ) (Fig. 2 and Table II). At the same time, the ROC curve shows that chemerin is a specific and sensitive biomarker for mild AoV stenosis (AUC $=0.82$; 0.80-0.90=good; $\mathrm{P}<0.001$ ) (Fig. 3 and Table III). The optimal cut-off value for chemerin in the ROC curve was $43.12 \mathrm{ng} / \mathrm{ml}$ at $\mathrm{Se}=87 \%, \mathrm{Sp}=69 \%$. FGF-21 is a poor diagnostic marker among all AoV stenosis groups without grading the severity $(\mathrm{AUC}=0.67 ; 0.56-0.77=$ poor $; \mathrm{P}=0.003)($ Fig .2 and Table II $)$ and in the mild AoV stenosis group $(\mathrm{AUC}=0.66 ; 0.51-0.81=$ poor; $\mathrm{P}=0.04$ ) (Fig. 3 and Table III).

\section{Discussion}

The results of the present study indicate that chemerin is a novel biomarker for the detection of AoV stenosis in the early stages, with a high sensitivity and specificity in the mild AoV stenosis group. Chemerin is found in different inflammatory and systemic processes all over the body (13,30,44-51): Therefore, patients with systemic and inflammatory diseases were excluded from this study. Thus, in the early stages of AoV stenosis, an active inflammation process is dominant, as chemerin is mainly considered an inflammatory biomarker $(16,20,32)$ and in our study its level was the highest in mild AoV stenosis. There is a possibility that chemerin is secreted due to the activation of valvular myofibrils, although there is a lack of evidence to support this theory and further research should be conducted. In more severe stages, the serum chemerin level decreased, leading to the conclusion that passive extracellular matrix changes and calcification predominate in moderate and severe calcific AoV stenosis. The ROC analysis proves that chemerin sensitivity and specificity as a diagnostic biomarker in mild AoV stenosis is high; thus, this test may be engrained in cardiovascular risk panels for laboratory analysis. Early detection of AoV stenosis may improve prognosis and quality of life of patients, improve therapy efficiency, and help to avoid late compensatory complications of AoV stenosis, such as cardiac hypertrophy. It would be useful to study other biomarkers that represent the inflammation process in AoV stenosis and find appropriate medications for treatment. Along with other studies mentioned above (21-27), our study does not state whether chemerin has a pro- or anti-inflammatory role and further research should be conducted with other biomarkers that support varying theories.

Of note is that the FGF-21 level increases along with the AoV stenosis severity grade. These results have led to the conclusion that FGF-21 plays a protective role in calcific AoV stenosis pathogenesis, because in the initial stages only a slight increase in plasma FGF-21 was evident, but in the more severe grade of AoV stenosis, it significantly increased. It has been suggested that FGF-21 may have a cardioprotective role in different cardiovascular diseases such as atrial fibrillation, myocardial infarction, coronary artery disease, hypertension, and cardiac hypertrophy, which supports our findings $(38,42)$.

In conclusion, our study indicates that chemerin is a good diagnostic biomarker in mild AoV stenosis and FGF-21 is a poor diagnostic biomarker for AoV stenosis. The present data show that the inflammatory process is dominant at the early stages of stenosis, which leads us to the conclusion that it is an active process not caused by atherosclerosis. Chemerin as a biomarker may play a clinical role if we can prove that it is one of the causes of inflammation; therefore, a possible treatment may be found. FGF-21 undergoes a marked increase in severe AoV stenosis compared to healthy controls, and it may have a cardioprotective role. Further research is necessary to confirm these findings and to specify what pathogenetic role these markers play in AoV stenosis.

\section{References}

1. Akerström F, Barderas MG and Rodríguez-Padial L: Aortic stenosis: A general overview of clinical, pathophysiological and therapeutic aspects. Expert Rev Cardiovasc Ther 11: 239-250, 2013.

2. Bossé Y, Mathieu P and Pibarot P: Genomics: The next step to elucidate the etiology of calcific aortic valve stenosis. J Am Coll Cardiol 51: 1327-1336, 2008.

3. Wypasek E, Potaczek DP and Undas A: Association of the C-reactive protein gene (CRP) rs1205 C $>\mathrm{T}$ polymorphism with aortic valve calcification in patients with aortic stenosis. Int J Mol Sci 16: 23745-23759, 2015.

4. Coffey S, Cox B and Williams MJA: The prevalence, incidence, progression, and risks of aortic valve sclerosis: A systematic review and meta-analysis. J Am Coll Cardiol 63: 2852-2861, 2014.

5. Carabello BA: Aortic stenosis: A fatal disease with but a single cure. JACC Cardiovasc Interv 1: 127-128, 2008.

6. Novaro GM and Griffin BP: Calcific aortic stenosis: Another face of atherosclerosis? Cleve Clin J Med 70: 471-477, 2003.

7. Fondard O, Detaint D, Iung B, Choqueux C, Adle-Biassette H, Jarraya M, Hvass U, Couetil JP, Henin D, Michel JB, et al: Extracellular matrix remodelling in human aortic valve disease: The role of matrix metalloproteinases and their tissue inhibitors. Eur Heart J 26: 1333-1341, 2005.

8. Galante A, Pietroiusti A, Vellini M, Piccolo P, Possati G, De Bonis M, Grillo RL, Fontana $\mathrm{C}$ and Favalli C: C-reactive protein is increased in patients with degenerative aortic valvular stenosis. J Am Coll Cardiol 38: 1078-1082, 2001.

9. Fayad ZA, Mani V, Woodward M, Kallend D, Abt M, Burgess T, Fuster V, Ballantyne CM, Stein EA, Tardif JC, et al: dal-PLAQUE Investigators: Safety and efficacy of dalcetrapib on atherosclerotic disease using novel non-invasive multimodality imaging (dal-PLAQUE): A randomised clinical trial. Lancet 378: 1547-1559, 2011.

10. Duvic M, Nagpal S, Asano AT and Chandraratna RAS: Molecular mechanisms of tazarotene action in psoriasis. J Am Acad Dermatol 37: S18-S24, 1997.

11. Roh SG, Song S-H, Choi K-C, Katoh K, Wittamer V, Parmentier M and Sasaki S: Chemerin-a new adipokine that modulates adipogenesis via its own receptor. Biochem Biophys Res Commun 362: 1013-1018, 2007.

12. Wittamer V, Franssen J-D, Vulcano M, Mirjolet J-F, Le Poul E, Migeotte I, Brézillon S, Tyldesley R, Blanpain C, Detheux M, et al: Specific recruitment of antigen-presenting cells by chemerin, a novel processed ligand from human inflammatory fluids. J Exp Med 198: 977-985, 2003.

13. Döcke S, Lock JF, Birkenfeld AL, Hoppe S, Lieske S, Rieger A, Raschzok N, Sauer IM, Florian S, Osterhoff MA, et al: Elevated hepatic chemerin mRNA expression in human non-alcoholic fatty liver disease. Eur J Endocrinol 169: 547-557, 2013. 
14. Weigert J, Neumeier M, Wanninger J, Filarsky M, Bauer S, Wiest R, Farkas S, Scherer MN, Schäffler A, Aslanidis C, et al: Systemic chemerin is related to inflammation rather than obesity in type 2 diabetes. Clin Endocrinol (Oxf) 72: 342-348, 2010.

15. Rourke JL, Dranse HJ and Sinal CJ: Towards an integrative approach to understanding the role of chemerin in human health and disease. Obes Rev 14: 245-262, 2013.

16. Lehrke M, Becker A, Greif M, Stark R, Laubender RP, von Ziegler F, Lebherz C, Tittus J, Reiser M, Becker C, et al: Chemerin is associated with markers of inflammation and components of the metabolic syndrome but does not predict coronary atherosclerosis. Eur J Endocrinol 161: 339-344, 2009.

17. Eisinger K, Krautbauer S, Wiest R, Weiss TS and Buechler C: Reduced serum chemerin in patients with more severe liver cirrhosis. Exp Mol Pathol 98: 208-213, 2015.

18. Gu P, Jiang W, Lu B and Shi Z: Chemerin is associated with inflammatory markers and metabolic syndrome phenotypes in hypertension patients. Clin Exp Hypertens 36: 326-332, 2014.

19. Kostopoulos CG, Spiroglou SG, Varakis JN, Apostolakis E and Papadaki HH: Chemerin and CMKLR1 expression in human arteries and periadventitial fat: A possible role for local chemerin in atherosclerosis? BMC Cardiovasc Disord 14: 56, 2014.

20. Goralski KB, McCarthy TC, Hanniman EA, Zabel BA, Butcher EC, Parlee SD, Muruganandan S and Sinal CJ: Chemerin, a novel adipokine that regulates adipogenesis and adipocyte metabolism. J Biol Chem 282: 28175-28188, 2007.

21. Bondue B, De Henau O, Luangsay S, Devosse T, de Nadaï P, Springael JY, Parmentier $M$ and Vosters O: The chemerin/ChemR23 system does not affect the pro-inflammatory response of mouse and human macrophages ex vivo. PLoS One 7: e40043, 2012.

22. Bondue B, Wittamer V and Parmentier M: Chemerin and its receptors in leukocyte trafficking, inflammation and metabolism. Cytokine Growth Factor Rev 22: 331-338, 2011.

23. Ernst MC and Sinal CJ: Chemerin: At the crossroads of inflammation and obesity. Trends Endocrinol Metab 21: 660-667, 2010

24. Hart R and Greaves DR: Chemerin contributes to inflammation by promoting macrophage adhesion to VCAM-1 and fibronectin through clustering of VLA-4 and VLA-5. J Immunol 185: 3728-3739, 2010

25. Kaur J, Adya R, Tan BK, Chen J and Randeva HS: Identification of chemerin receptor (ChemR23) in human endothelial cells: Chemerin-induced endothelial angiogenesis. Biochem Biophys Res Commun 391: 1762-1768, 2010.

26. Monnier J, Lewén S, O'Hara E, Huang K, Tu H, Butcher EC and Zabel BA: Expression, regulation, and function of atypical chemerin receptor CCRL2 on endothelial cells. J Immunol 189: 956-967, 2012

27. Zhao RJ and Wang $\mathrm{H}$ : Chemerin/ChemR23 signaling axis is involved in the endothelial protection by K(ATP) channel opener iptakalim. Acta Pharmacol Sin 32: 573-580, 2011

28. Kukla M, Zwirska-Korczala K, Gabriel A, Waluga M, Warakomska I, Szczygiel B, Berdowska A, Mazur W, Wozniak-Grygiel E and Kryczka W: Chemerin, vaspin and insulin resistance in chronic hepatitis C. J Viral Hepat 17: 661-667, 2010

29. Cash JL, Hart R, Russ A, Dixon JPC, Colledge WH, Doran J, Hendrick AG, Carlton MB and Greaves DR: Synthetic chemerin-derived peptides suppress inflammation through ChemR23. J Exp Med 205: 767-775, 2008.

30. Mariani F and Roncucci L: Chemerin/chemR23 axis in inflammation onset and resolution. Inflamm Res 64: 85-95, 2015.

31. Semba RD, Crasto C, Strait J, Sun K, Schaumberg DA and Ferrucci L: Elevated serum fibroblast growth factor 21 is associated with hypertension in community-dwelling adults. J Hum Hypertens 27: 397-399, 2013.

32. Van de Voorde J, Pauwels B, Boydens C and Decaluwé K: Adipocytokines in relation to cardiovascular disease. Metabolism 62: 1513-1521, 2013.

33. Zhang X, Yeung DCY, Karpisek M, Stejskal D, Zhou ZG, Liu F, Wong RL, Chow WS, Tso AW, Lam KS, et al: Serum FGF21 levels are increased in obesity and are independently associated with the metabolic syndrome in humans. Diabetes 57: 1246-1253, 2008

34. An SY, Lee MS, Yi SA, Ha ES, Han SJ, Kim HJ, Kim DJ and Lee KW: Serum fibroblast growth factor 21 was elevated in subjects with type 2 diabetes mellitus and was associated with the presence of carotid artery plaques. Diabetes Res Clin Pract 96: 196-203, 2012
35. Chow WS, Xu A, Woo YC, Tso AWK, Cheung SCW, Fong CHY, Tse HF, Chau MT, Cheung BM and Lam KS: Serum fibroblast growth factor-21 levels are associated with carotid atherosclerosis independent of established cardiovascular risk factors. Arterioscler Thromb Vasc Biol 33: 2454-2459, 2013.

36. Lin Z, Wu Z, Yin X, Liu Y, Yan X, Lin S, Xiao J, Wang X, Feng $W$ and Li X: Serum levels of FGF-21 are increased in coronary heart disease patients and are independently associated with adverse lipid profile. PLoS One 5: e15534, 2010.

37. Xu P, Zhang Y, Liu Y, Yuan Q, Song L, Liu M, Liu Z, Yang Y, Li J, Li D, et al: Fibroblast growth factor 21 attenuates hepatic fibrogenesis through TGF- $\beta / \mathrm{smad} 2 / 3$ and NF- $\kappa \mathrm{B}$ signaling pathways. Toxicol Appl Pharmacol 290: 43-53, 2016.

38. Han X, Chen C, Cheng G, Xie C, Yang M, Shou X and Sun C: Serum fibroblast growth factor 21 levels are increased in atrial fibrillation patients. Cytokine 73: 176-180, 2015

39. Itoh $\mathrm{N}$ and Ornitz DM: Fibroblast growth factors: From molecular evolution to roles in development, metabolism and disease. J Biochem 149: 121-130, 2011

40. Patel V, Adya R, Chen J, Ramanjaneya M, Bari MF, Bhudia SK, Hillhouse EW, Tan BK and Randeva HS: Novel insights into the cardio-protective effects of FGF21 in lean and obese rat hearts. PLoS One 9: e87102, 2014

41. Planavila A, Redondo I, Hondares E, Vinciguerra M, Munts C, Iglesias R, Gabrielli LA, Sitges M, Giralt M, van Bilsen M, and Villarroya F: Fibroblast growth factor 21 protects against cardiac hypertrophy in mice. Nat Commun 4:2019, 06 2013. doi: 10.1038/ ncomms3019.

42. Planavila A, Redondo-Angulo I and Villarroya F: FGF21 and cardiac physiopathology. Front Endocrinol 6: 133, 2015.

43. Vahanian A and Iung B: The new ESC/EACTS guidelines on the management of valvular heart disease. Arch Cardiovasc Dis 105: 465-467, 2012

44. Gisondi P, Lora V, Bonauguri C, Russo A, Lippi G and Girolomoni G: Serum chemerin is increased in patients with chronic plaque psoriasis and normalizes following treatment with infliximab. Br J Dermatol 168: 749-755, 2013.

45. Herenius MMJ, Oliveira ASF, Wijbrandts CA, Gerlag DM, Tak PP and Lebre MC: Anti-TNF therapy reduces serum levels of chemerin in rheumatoid arthritis: A new mechanism by which anti-TNF might reduce inflammation. PLoS One 8: e57802, 2013.

46. Makrilakis K, Fragiadaki K, Smith J, Sfikakis PP and Kitas GD: Interrelated reduction of chemerin and plasminogen activator inhibitor-1 serum levels in rheumatoid arthritis after interleukin-6 receptor blockade. Clin Rheumatol 34: 419-427, 2015.

47. Rutkowski P, Sledzinski T, Zielinska H, Lizakowski S, Goyke E, Szrok-Wojtkiewicz S, Swierczynski J and Rutkowski B: Decrease of serum chemerin concentration in patients with end stage renal disease after successful kidney transplantation. Regul Pept 173: $55-59,2012$

48. Stepan H, Philipp A, Roth I, Kralisch S, Jank A, Schaarschmidt W, Lössner U, Kratzsch J, Blüher M, Stumvoll M, et al: Serum levels of the adipokine chemerin are increased in preeclampsia during and 6 months after pregnancy. Regul Pept 168: 69-72, 2011.

49. Weigert J, Obermeier F, Neumeier M, Wanninger J, Filarsky M, Bauer S, Aslanidis C, Rogler G, Ott C, Schäffler A, et al: Circulating levels of chemerin and adiponectin are higher in ulcerative colitis and chemerin is elevated in Crohn's disease. Inflamm Bowel Dis 16: 630-637, 2010.

50. Xu QL, Zhu M, Jin Y, Wang N, Xu HX, Quan LM, Wang SS and Li SS: The predictive value of the first-trimester maternal serum chemerin level for pre-eclampsia. Peptides 62: 150-154, 2014.

51. Zhang J, Jin HC, Zhu AK, Ying RC, Wei W and Zhang FJ: Prognostic significance of plasma chemerin levels in patients with gastric cancer. Peptides 61: 7-11, 2014.

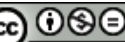

This work is licensed under a Creative Common Attribution-NonCommercial-NoDerivatives 4.0 International (CC BY-NC-ND 4.0) License. 